\title{
A Novel Method for Classifying Body Mass Index on the Basis of Speech Signals for Future Clinical Applications: A Pilot Study
}

\author{
Bum Ju Lee, Boncho Ku, Jun-Su Jang, and Jong Yeol Kim \\ Medical Research Division, Korea Institute of Oriental Medicine, 1672 Yuseongdae-ro, Yuseong-gu, \\ Deajeon 305-811, Republic of Korea \\ Correspondence should be addressed to Jong Yeol Kim; ssmed@kiom.re.kr
}

Received 5 November 2012; Revised 11 January 2013; Accepted 13 January 2013

Academic Editor: Hyunsu Bae

Copyright (C) 2013 Bum Ju Lee et al. This is an open access article distributed under the Creative Commons Attribution License, which permits unrestricted use, distribution, and reproduction in any medium, provided the original work is properly cited.

Obesity is a serious public health problem because of the risk factors for diseases and psychological problems. The focus of this study is to diagnose the patient BMI (body mass index) status without weight and height measurements for the use in future clinical applications. In this paper, we first propose a method for classifying the normal and the overweight using only speech signals. Also, we perform a statistical analysis of the features from speech signals. Based on 1830 subjects, the accuracy and AUC (area under the ROC curve) of age- and gender-specific classifications ranged from 60.4 to $73.8 \%$ and from 0.628 to 0.738 , respectively. We identified several features that were significantly different between normal and overweight subjects $(P<0.05)$. Also, we found compact and discriminatory feature subsets for building models for diagnosing normal or overweight individuals through wrapper-based feature subset selection. Our results showed that predicting BMI status is possible using a combination of speech features, even though significant features are rare and weak in age- and gender-specific groups and that the classification accuracy with feature selection was higher than that without feature selection. Our method has the potential to be used in future clinical applications such as automatic BMI diagnosis in telemedicine or remote healthcare.

\section{Introduction}

Worldwide, increasing numbers of people are becoming obese, including adults, adolescents, and children and both men and woman $[1,2]$. Obesity refers to excess adipose tissue caused by genetic determinants, excessive eating, insufficient physical movement, and an inappropriate lifestyle $[1,3,4]$. Obesity and being overweight are serious public health problems; obesity has a direct relationship with physical health and psychological health and is a potential risk factor for many diseases, including cardiovascular diseases, stroke, ischemic heart disease, diabetes, and cancer $[2,5-$ 8]. Therefore, it is important to recognize when patients are overweight or obese, and many studies have been performed about the relationship of obesity, as determined by body mass index (BMI), and disease [4, 6, 7, 9-11]. BMI, proposed by Lambert Adolphe Jacques Quetelet, is a measurement criterion presenting the relationship between body weight and height [3] and a commonly used public health method for classifying underweight, normal, overweight, and obese patients.

On the other hand, research on the association of body shape (weight, height), age, and gender with speech signals has been conducted over a long period in various fields such as speech recognition, security technology, and forensic and medical science, and many studies have suggested a strong or weak relationship between body shape and speech signals [12-28]. Previous analysis of body shape and speech signals has determined that there are differences between normal and obese people in terms of the facial skeleton, the function of the upper airway, and the surrounding structure of the upper airway [12], and that there is a significant association of body shape with vocal tract length [13]. In various vocal features, the fundamental frequency (pitch) of men was associated with measurements of body shape and size such as chest circumference and shoulder-hip ratio [14]. In more detail, Evans et al. suggested that the fundamental frequency in men is an indicator of body configuration based on their findings 
of a significant association of large body shape with low fundamental frequency and a significantly negative correlation between weight and fundamental frequency [14]. Lass et al. $[15,16]$ showed a relationship among heights, weights, body surface areas, and fundamental frequencies of a speaker using Pearson correlation coefficients, and they suggested acoustic cues for accurate estimation of speaker height and weight. van Dommelen and Moxness [17] investigated the ability of listeners to determine the weight and height of speakers using average fundamental frequency, energy below $1 \mathrm{kHz}$, and speech rate. Although they did not find any significant correlations between these features and the height or weight of the speaker, they suggested that speech rate is a good predictor of the weight of male speakers. González [18] examined the relationship between formant frequencies and the height and weight of subjects aged 20 to 30 years in Spain and reported a weak relationship between body size and formant frequencies in adults of the same gender; moreover, the relationship was stronger in women than in men. His results contradicted those of Fitch, who reported a strong correlation between body size and formant dispersions in macaques. Furthermore, Künzel [19] analyzed the relationship between average fundamental frequency and the weight and height of subjects in Wiesbaden, Germany, but found no significant correlations between vocal features and weight or height. Meanwhile, in previous studies of the association of gender, age, and cultural factor with speech signals, Childers and $\mathrm{Wu}$ [20] studied the automatic recognition of gender using vocal features and found that the second formant frequency is a slightly better predictor of gender than the fundamental frequency. Bruckert et al. [21] investigated the reliability of vocal features as indicators of a speaker's physical features and found that men with small formant dispersion and lowfrequency formants tend to be taller and older and have high testosterone levels. They argue that cultural factors must be considered when determining correlations between speakers' height and weight and vocal features. Similarly, Belin et al. [22] argue that vocal habits, cultural factors, and age and gender differences play important roles in shaping voice quality. In addition, forensic speaker classifications in the domains of dialect, foreign accent, sociolect, age, gender, and medical conditions were well summarized by Jessen [23], who stated that auditory and acoustic analyses are essential works for forensic speaker classification.

In this study, we ask whether it is possible to classify BMI status of patients using only voice information. If it is possible to know a patient's BMI category on the basis of voice data-irrespective of height and weight informationthis can be used as alternative or subsidiary information for the diagnosis of normal weight or obesity and for prognosis prediction, under the assumption of circumstances such as remote medical care environments and real-time monitoring services to support general treatment or emergency medical services. For example, BMI values are calculated from weight and height $\left(\mathrm{kg} / \mathrm{m}^{2}\right)$. Thus, to get the BMI value of patients or potential patients, weight and height must be measured on the spot. However, these measurements are sometimes not suitable for remote healthcare or $\mathrm{u}$-healthcare supporting general treatment and emergency medical service in real time at remote locations, since $22 \%$ of patients do not estimate their own weight within $\pm 5 \mathrm{~kg}$, even though patient selfestimates of weight are better than estimates by residents and nurses in emergency department [29]. In remote medicine for real-time communication in remote locations, many patients do not know their exact weight at the time of diagnosis of BMI because the weight of patients was changed slowly or rapidly over time. We must obtain the maximal clinic data of patients rapidly and often with minimal network or telephone time and communication equipment. Because a great deal of medical information is needed for patient care and prognosis prediction $[30,31]$, telemedicine or remote healthcare system facilitates the quality and quantity of data collection and integration, communication between patients and healthcare systems, preprocessing to optimize medical treatment, and decision support and modification of medical treatment primarily using telephones, computers, fax, and WCU VC (virtual community program) [32-34]. Also, the technologies have the advantages of health improvement, patient convenience, cost effectiveness, economy of time, data accuracy and permanence, and continuous real-time monitoring of chronic disease [35-37].

Our contributions in this study are as follows: we first propose a method for classifying the normal weight or the high weight using speech signals in age-and genderspecific groups. Our method may apply to the development of advanced and automatic methods for individual BMI diagnosis in telemedicine and u-healthcare and assist in the development of a simpler system for BMI measurement. Also, our suggestion that is possible to support context awareness may provide clues to improve the overall quality of emergency service via automatic support of patient BMI information in remote healthcare systems with limited resources. We find discriminatory and meaningful features for normal and overweight diagnoses via a statistical analysis between BMI and speech features and identify a compact and useful feature subset in accordance with the age-and gender-specific analysis. The results will serve to create a better discriminatory feature set and accurate classification models in this field.

\section{Materials and Methods}

\subsection{Data Preparation}

2.1.1. Data Collection. A total of 1830 people participated in this study. Data was collected from subjects in several hospitals and the Korea Institute of Oriental Medicine in the Republic of Korea. Subjects with any voice-related diseases were excluded from this study. Speech recording configurations were as follows: no resonance; room temperature, $20^{\circ} \mathrm{C}$ $\left( \pm 5^{\circ} \mathrm{C}\right)$; noise intensity, $<40 \mathrm{~dB}$; and humidity, $40 \%( \pm 5 \%)$. Personal computers and an external sound card (Blaster Live 24-bit) to avoid noise from the personal computers were used for initial voice acquisition. GoldWave v5.58 was used to record audio data, and the voice files were saved in the wav format. The distance from the subjects' mouth to the microphone (Sennheiser e-835s microphone) was $4-6 \mathrm{~cm}$. 
The recording of the speakers' speech was strictly controlled by a standard operating procedure (SOP). The SOP was established to capture the natural characteristics of the speakers in short recordings. The speakers rested for 1 hour before actual recording to reduce suspense. An operator instructed the speakers regarding the recording content, and the speakers were asked to pronounce words in their normal tone without tension. The operator constantly monitored the speakers' speech and their distance from the microphone while recording. When the speakers could not produce a uniform tone for 5 vowels, their speech was rerecorded until they achieved a certain level of tone uniformity. Each sentence was recorded twice, and the value of each feature was obtained by averaging the values of the 2 recordings for more stable features.

All features were extracted using 5 vowels (A, E, I, $\mathrm{O}, \mathrm{U}$ ) and 1 sentence [38]. For speech feature extraction, we extracted 65 features from the collected data set. The extracted features consisted of pitch, average ratio of pitch period, correlation coefficient between F0 and intensity (CORR), absolute Jitter (Jita), and Mel frequency cepstral coefficients (MFCC), among others [18, 23, 27]. The specific content of the extracted features is described in Table 1 , and sample of speech signal recording of 5 vowels and one sentence is showed in Figure 1.

2.1.2. Class Label Decision for Normal and Overweight Statuses. Obesity and BMI research is difficult due to different ethnic groups and different national economic statuses [7]. Also, BMI values differ according to physiological factors and environmental factors, such as residing in a city or a rural area. For instance, BMI values of a population in an Asian region tend to be lower than those of a population in a Western region, whereas Asians have risk factors for cardiovascular disease and diabetes related to obesity at relatively low BMI values $[9,39]$. The BMI cutoff values for overweight and obesity depend on several factors including ethnicity, rural/urban residence, and economic status $[7,40]$. Therefore, we decided that this study's overweight cutoff point of BMI value was $\geq 23 \mathrm{~kg} / \mathrm{m}^{2}$, according to suggestions by the World Health Organization and references [39, 41, 42]. We refer here to only 2 classes: the "normal" and the "overweight." Subjects in the BMI who range from 18.5 to 22.9 were labeled normal, and subjects with a BMI of 23 or over were labeled as overweight. Underweight patients were passed over due the lack of a minimum number of subjects. Finally, we divided the data set into 6 groups for age-and gender-specific classification: female: $20-30$ (females aged $20-39$ years), female: $40-50$ (females aged $40-59$ years), female: 60 (females aged 60 years and over), male: 20-30 (males aged 20-39 years), male: 40-50 (males aged 40-59 years), and male: 60 (males aged 60 years and over).

The overall mean ages of the female and male subjects were 41.79 and 40.51 , respectively. The mean age and standard deviation of females aged 20-39 years were 28.22 and \pm 6.326 , and the mean BMI and standard deviation were 21.76 and \pm 2.489 . The rest of the groups are described in Table 2. The number of normal and overweight subjects in the 6 groups is described in Table 4.

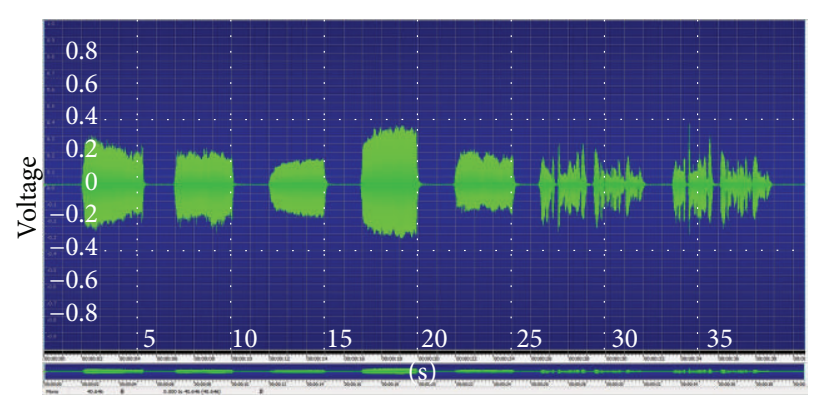

(a)

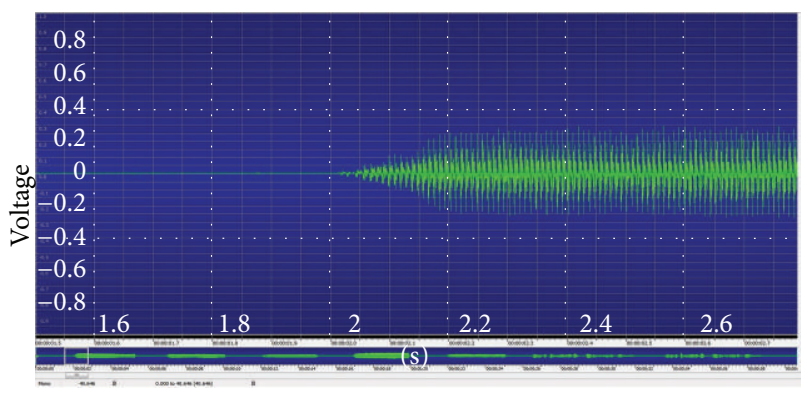

(b)

FIGURE 1: Sample of speech signal recording of 5 vowels and one sentence ((a): signals of 5 vowels and one sentence and (b): detailed signal of one vowel to demonstrate the difference between noise and signal).

2.2. Feature Selection and Experiment Configurations. For feature subset selection, we applied normalization (scale $0 \sim 1$ value) to all data sets. The Wrapper-based feature selection approach $[43,44]$ using machine learning of logistic regression $[30,45]$ with genetic search was used to maximize the area under ROC curve (AUC). The selected features in each group are shown in Table 3. All experiments were performed using logistic regression in Weka [46], and a 10-fold cross validation was performed [47]. We used the accuracy, true positive rate (sensitivity, TPR), false positive rate (1 specificity, FPR), precision, and F measure as performance evaluation criteria $[47,48]$. A large proportion of classification algorithms may not solve the class-size imbalance problem [49]. Thus, the accuracy of many classification experiments is higher for a majority class than for a minority class. Therefore, we also evaluated performance using AUC. An ROC curve (receiver operating characteristic curve) represents the balance of sensitivity versus 1 specificity [50]. Because the AUC is a threshold-independent measure, AUC is a widely used to quantify the quality of a prediction or classification model in medical science, bioinformatics, medicine statistics, and biology [31, 51-53]. An AUC of 1 means a perfect diagnosis model, an AUC of 0.5 is random diagnosis, and an AUC of 0 is a perfectly wrong diagnosis.

\section{Results and Discussion}

Our experiments were divided into two steps. In the first experiment, we conducted classification of normal and overweight classes with six data sets according to age-and 
TABLE 1: All features used in this study and brief descriptions.

\begin{tabular}{|c|c|c|c|}
\hline Feature & Brief description & Feature & Brief description \\
\hline $\mathrm{aF0}$ & Basic pitch of $\mathrm{A}$ & oPPQ & Smoothing value around JITA of $\mathrm{O}$ \\
\hline aJITA & Mean ratio of change in pitch period of $\mathrm{A}$ & oF60_120_240_480 & $\begin{array}{l}(\text { energy of } 60 \sim 120 \mathrm{~Hz}) /(\text { energy of } \\
240 \sim 480 \mathrm{~Hz}) \text { of } \mathrm{O}\end{array}$ \\
\hline aJITT & Percentage of JITA value of A & oF240_480_960_1920 & $\begin{array}{l}\text { (energy of } 240 \sim 480 \mathrm{~Hz}) /(\text { energy of } \\
960 \sim 1920 \mathrm{~Hz} \text { ) of O }\end{array}$ \\
\hline aPPQ & Smoothing value around JITA of A & oF60_120_oF960_1920 & $\begin{array}{l}(\text { energy of } 60 \sim 120 \mathrm{~Hz}) /(\text { energy of } \\
960 \sim 1920 \mathrm{~Hz}) \text { of } \mathrm{O}\end{array}$ \\
\hline aF60_120_F240_480 & $\begin{array}{l}\text { (energy of } 60 \sim 120 \mathrm{~Hz}) /(\text { energy of } \\
240 \sim 480 \mathrm{~Hz} \text { ) of A }\end{array}$ & $\mathrm{oF} 1$ & Formant of first in 4 frequency periods of $\mathrm{O}$ \\
\hline aF240_480_960_1920 & $\begin{array}{l}\text { (energy of } 240 \sim 480 \mathrm{~Hz}) /(\text { energy of } \\
960 \sim 1920 \mathrm{~Hz} \text { ) of A }\end{array}$ & oF2 & $\begin{array}{l}\text { Formant of second in } 4 \text { frequency } \\
\text { periods of } \mathrm{O}\end{array}$ \\
\hline aF60_120_960_1920 & $\begin{array}{l}\text { (energy of } 60 \sim 120 \mathrm{~Hz}) /(\text { energy of } \\
960 \sim 1920 \mathrm{~Hz} \text { ) of A }\end{array}$ & oF2_F1 & Difference of frequencies (oF2-F1) \\
\hline $\mathrm{aF} 1$ & Formant of first in 4 frequency periods of $\mathrm{A}$ & $\mathrm{uF0}$ & Basic pitch of $U$ \\
\hline $\mathrm{aF} 2$ & Formant of second in 4 frequency periods of $\mathrm{A}$ & uJITA & Mean ratio of change in pitch period of $U$ \\
\hline aF2_F1 & $\mathrm{aF} 2 / \mathrm{F} 1$ & uJITT & Percentage of JITA value of $U$ \\
\hline eF0 & Basic pitch of $\mathrm{E}$ & uPPQ & Smoothing value around JITA of $U$ \\
\hline eJITA & Mean ratio of change in pitch period of $\mathrm{E}$ & uF60_120_240_480 & $\begin{array}{l}\text { (energy of } 60 \sim 120 \mathrm{~Hz}) /(\text { energy of } \\
240 \sim 480 \mathrm{~Hz} \text { ) of } \mathrm{U}\end{array}$ \\
\hline eJITT & Percentage of JITA value of E & uF240_480_960_1920 & $\begin{array}{l}\text { (energy of } 240 \sim 480 \mathrm{~Hz}) /(\text { energy of } \\
960 \sim 1920 \mathrm{~Hz} \text { ) of } \mathrm{U}\end{array}$ \\
\hline ePPQ & Smoothing value around JITA of E & uF60_120_960_1920 & $\begin{array}{l}\text { (energy of } 60 \sim 120 \mathrm{~Hz}) /(\text { energy of } \\
960 \sim 1920 \mathrm{~Hz} \text { ) of } \mathrm{U}\end{array}$ \\
\hline eF60_120_240_480 & $\begin{array}{l}\text { (energy of } 60 \sim 120 \mathrm{~Hz}) /(\text { energy of } \\
240 \sim 480 \mathrm{~Hz} \text { ) of } \mathrm{E}\end{array}$ & $\mathrm{uF} 1$ & Formant of first in 4 frequency periods of $U$ \\
\hline eF240_480_960_1920 & $\begin{array}{l}\text { (energy of } 240 \sim 480 \mathrm{~Hz}) /(\text { energy of } \\
960 \sim 1920 \mathrm{~Hz} \text { ) of } \mathrm{E}\end{array}$ & $\mathrm{uF} 2$ & $\begin{array}{l}\text { Formant of second in } 4 \text { frequency } \\
\text { periods of } U\end{array}$ \\
\hline eF60_120_960_1920 & $\begin{array}{l}(\text { energy of } 60 \sim 120 \mathrm{~Hz}) /(\text { energy of } \\
960 \sim 1920 \mathrm{~Hz} \text { ) of } \mathrm{E}\end{array}$ & uF2_F1 & Difference of frequencies (uF2-F1) \\
\hline $\mathrm{eF} 1$ & Formant of first in 4 frequency periods of $\mathrm{E}$ & iF0_aF0 & Difference of frequencies (iF0-aF0) \\
\hline eF2 & Formant of second in 4 frequency periods of $\mathrm{E}$ & uF0_oF0 & Difference of frequencies ( $\mathrm{uF0}-\mathrm{oF} 0$ ) \\
\hline eF2_F1 & Difference of frequencies (eF2-F1) & aMFCC4 & $\begin{array}{l}\text { The terms of Mel frequency cepstral } \\
\text { coefficients of A }\end{array}$ \\
\hline $\mathrm{iF} 0$ & Basic pitch of I & eMFCC4 & $\begin{array}{l}\text { The terms of Mel frequency cepstral } \\
\text { coefficients of E }\end{array}$ \\
\hline iJITA & Mean ratio of change in pitch period of I & iMFCC4 & $\begin{array}{l}\text { The terms of Mel frequency cepstral } \\
\text { coefficients of I }\end{array}$ \\
\hline iJITT & Percentage of JITA value of I & oMFCC4 & $\begin{array}{l}\text { The terms of Mel frequency cepstral } \\
\text { coefficients of } \mathrm{O}\end{array}$ \\
\hline iPPQ & Smoothing value around JITA of I & uMFCC4 & $\begin{array}{l}\text { The terms of Mel frequency cepstral } \\
\text { coefficients of } U\end{array}$ \\
\hline iF60_120_240_480 & $\begin{array}{l}\text { (energy of } 60 \sim 120 \mathrm{~Hz}) /(\text { energy of } \\
240 \sim 480 \mathrm{~Hz} \text { ) of I }\end{array}$ & CORR & $\begin{array}{l}\text { Pearson correlation coefficients between F0 } \\
\text { and intensity }\end{array}$ \\
\hline iF240_480_960_1920 & $\begin{array}{l}\text { (energy of } 240 \sim 480 \mathrm{~Hz}) /(\text { energy of } \\
960 \sim 1920 \mathrm{~Hz} \text { ) of I }\end{array}$ & P50 & 50th percentile of F0 \\
\hline iF60_120_960_1920 & $\begin{array}{l}\text { (energy of } 60 \sim 120 \mathrm{~Hz}) /(\text { energy of } \\
960 \sim 1920 \mathrm{~Hz} \text { ) of I }\end{array}$ & $\mathrm{I} 50$ & 50th percentile of intensity \\
\hline $\mathrm{iF} 1$ & Formant of first in 4 frequency periods of I & SF0 & Mean pitch of sentence \\
\hline $\mathrm{iF} 2$ & Formant of second in 4 frequency periods of I & SSTD & Standard deviation of mean pitch of sentence \\
\hline iF2_F1 & Difference of frequencies (iF2-F1) & SITS & Intensity average \\
\hline oF0 & Basic pitch of $\mathrm{O}$ & SISTD & Standard deviation of intensity \\
\hline oJITA & Mean ratio of change in pitch period of $\mathrm{O}$ & SSPD & Time to read one sentence \\
\hline oJITT & Percentage of JITA value of $\mathrm{O}$ & Total & 65 \\
\hline
\end{tabular}


TABLE 2: Mean and standard deviation of age and BMI by each group.

\begin{tabular}{lcccccc}
\hline & Female: $20-30$ & Female: $40-50$ & Female: 60 & Male: $20-30$ & Male: $40-50$ & Male: 60 \\
\hline Age & $28.22 \pm 6.326$ & $48.7 \pm 5.555$ & $67.14 \pm 5.254$ & $27.34 \pm 5.433$ & $49.24 \pm 5.257$ & $66.75 \pm 4.995$ \\
BMI & $21.76 \pm 2.489$ & $23.76 \pm 3.048$ & $24.96 \pm 3.042$ & $23.71 \pm 2.971$ & $24.67 \pm 3.090$ & $23.59 \pm 2.3$ \\
\hline
\end{tabular}

TABLE 3: Selected features by feature selection in each group ( $N$ : number of selected features).

\begin{tabular}{|c|c|c|}
\hline Model (group) & $N$ & Selected features \\
\hline Female: $20-30$ & 25 & $\begin{array}{l}\text { aJITT, aPPQ, aF60_120_F240_480, aF240_480_960_1960, aF60_120_960_1960, aF1, eF0, eJITA, ePPQ, } \\
\text { eF240_480_960_1960, eF2, iPPQ, iF60_120_960_1960, oF0, oJITT, oF1, oF2, uF0, uJITT, aMFCC4, eMFCC4, } \\
\text { oMFCC4, uMFCC4, SF0, SITS }\end{array}$ \\
\hline Female: $40-50$ & 29 & $\begin{array}{l}\text { aF0, aJITA, aJITT, aF240_480_960_1960, aF2, eF0, eJITT, ePPQ, eF2_F1, iJITA, iPPQ, iF60_120_240_480, } \\
\text { iF240_480_960_1960, iF60_120_960_1960, oF0, oF240_480_960_1960, oF1, oF2, uF0, uPPQ, uF60_120_960_1960, } \\
\text { uF1, uF2, uF2_F1, aMFCC4, uMFCC4, CORR, I50, SISTD }\end{array}$ \\
\hline Female: 60 & 22 & $\begin{array}{l}\text { aJITA, aJITT, aF60_120_F240_480, aF240_480_960_1960, eJITT, ePPQ, eF240_480_960_1960, eF2_F1, } \\
\text { iF60_120_240_480, iF240_480_960_1960, iF60_120_960_1960, iF2, oF0, oJITT, oF2, oF2_F1, uF0, uJITA, } \\
\text { uF60_120_240_480, uF60_120_960_1960, uMFCC4, SISTD }\end{array}$ \\
\hline Male: $20-30$ & 8 & aJITA, aPPQ, eF2, iF1, oJITT, uPPQ, eMFCC4, uMFCC4 \\
\hline Male: $40-50$ & 24 & $\begin{array}{l}\text { aF0, eF0, eJITA, eJITT, eF60_120_960_1960, eF1, eF2, eF2_F1, iF0, iJITA, iPPQ, iF60_120_240_480, } \\
\text { iF240_480_960_1960, iF2, oJITA, oJITT, oPPQ, oF60_120_oF960_1960, oF1, oF2, uF0, uF60_120_960_1960, } \\
\text { eMFCC4, SF0 }\end{array}$ \\
\hline Male: 60 & 23 & $\begin{array}{l}\text { aJITT, aF60_120_F240_480, aF60_120_960_1960, aF1, eF240_480_960_1960, eF1, eF2_F1, iJITA, iPPQ, } \\
\text { iF240_480_960_1960, iF60_120_960_1960, iF1, iF2, iF2_F1, oF60_120_240_480, uF2, uF0_oF0, oMFCC4, P50, I50, } \\
\text { SSTD, SITS, SSPD }\end{array}$ \\
\hline
\end{tabular}

TABLE 4: Specific performance results (with feature selection, $N$ : number of subjects of each class).

\begin{tabular}{lcccccc}
\hline Model (group) & Class & $N$ & Sensitivity & False positive rate (1-specificity) & Precision & $F$ Measure \\
\hline \multirow{2}{*}{ Female: $20-30$} & Normal & 364 & 0.926 & 0.774 & 0.766 & 0.838 \\
& Overweight & 133 & 0.226 & 0.074 & 0.526 & 0.316 \\
Female: $40-50$ & Normal & 201 & 0.512 & 0.311 & 0.575 \\
& Overweight & 244 & 0.689 & 0.488 & 0.632 \\
Female: 60 & Normal & 41 & 0.366 & 0.173 & 0.455 \\
& Overweight & 104 & 0.827 & 0.634 & 0.768 & 0.659 \\
Male: $20-30$ & Normal & 175 & 0.52 & 0.325 & 0.576 \\
& Overweight & 206 & 0.675 & 0.48 & 0.623 \\
Male: $40-50$ & Normal & 77 & 0.377 & 0.14 & 0.547 \\
& Overweight & 179 & 0.86 & 0.623 & 0.648 \\
Male: 60 & Normal & 35 & 0.429 & 0.239 & 0.537 \\
& Overweight & 71 & 0.761 & 0.571 & 0.762 & 0.443 \\
\hline
\end{tabular}

gender-specific groups without feature selection. A goal of the experiment was to measure the ability to distinguish the normal and the overweight in each group using full features. Also, we want to identify a more compact and discriminatory feature set for detailed classification of each group. Therefore, in the second step, we applied a feature subset selection method to all data sets used in the first experiment. 12 classification models were built in the first and second steps.

3.1. Performance Evaluations. All of the performances in experiments applied to feature selection (FS-feature sets) in age- and gender-specific experiments were superior than those in experiments without feature selection (full-feature sets). Figures 2 and 3 show that the improvements in AUC and accuracy offered by feature selection were statistically significant. The accuracies for the 6 groups using full-feature sets ranged from 50.9 to $68.8 \%$. After feature selection, the accuracies for the 6 groups using FS-feature sets ranged from 60.4 to $73.8 \%$, and the average accuracy of the 6 groups improved by about $8.4 \%$ compared with the use of fullfeature sets. The highest accuracy among the groups was $73.8 \%$ (female: $20-30$ ), and the lowest accuracy was $60.4 \%$ (male: 20-30).

However, AUC results based on sensitivity and false positive rates (1 specificity) were slightly different from the accuracy results. AUC using FS-feature sets ranged from 


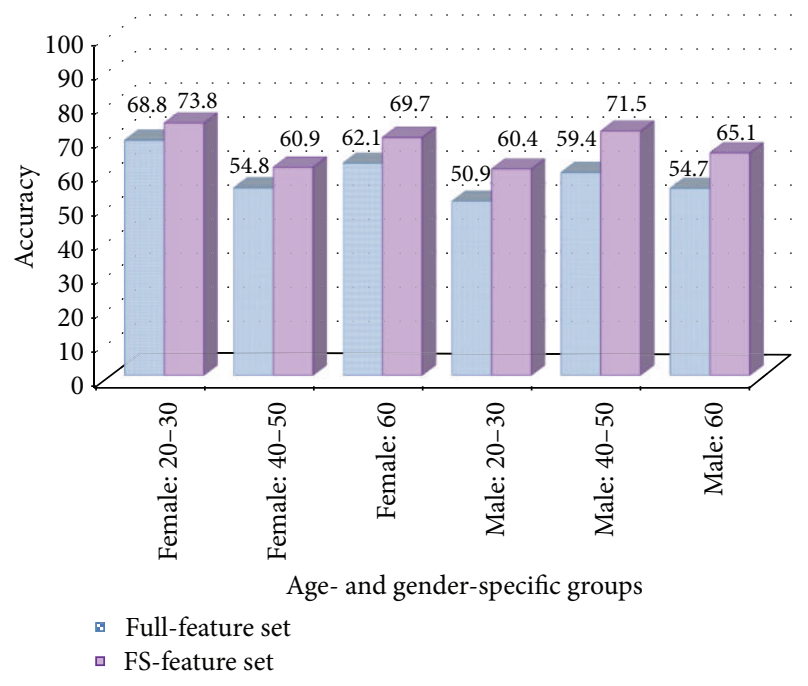

FIgURE 2: Accuracy comparison of experiment results between fullfeature set and FS-feature set in 6 groups.

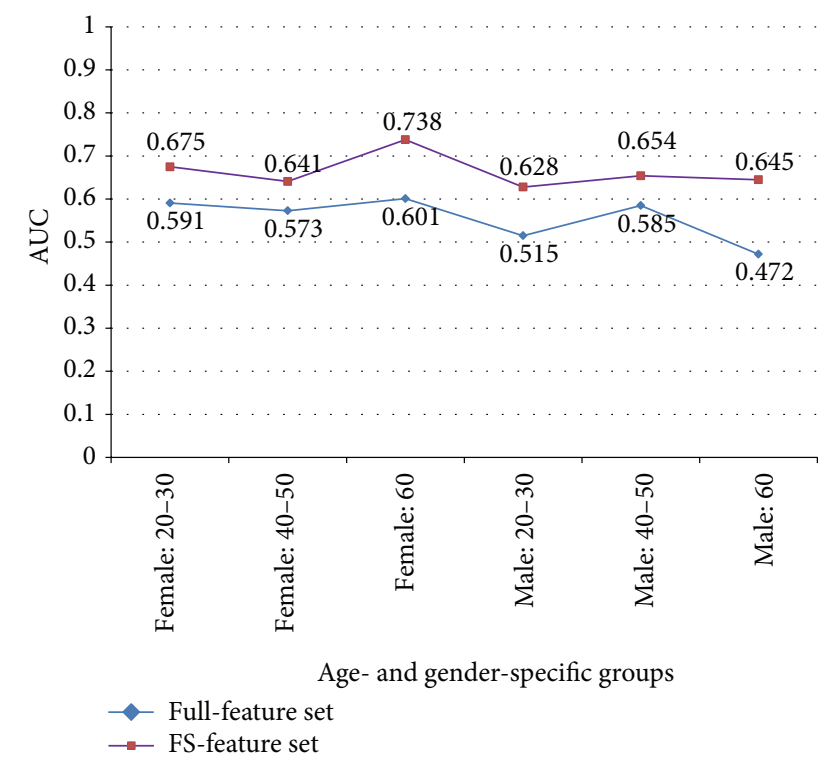

FIGURE 3: AUC comparison of experiment results between fullfeature set and FS-feature set in 6 groups.

0.628 to 0.738 . The accuracy of the female: 60 group was lower than that of female: $20-30$ and male: $40-50$, but the AUC of female: 60 was the highest among the 6 groups. The specific performance results of female: 60 using the FS-feature set included a sensitivity of $0.366, \mathrm{FPR}$ of 0.173 , precision of 0.455 , and $\mathrm{F}$ measure of 0.405 in the normal weight class and a sensitivity of 0.827 , FPR of 0.634 , precision of 0.768 , and $\mathrm{F}$ measure of 0.796 in the overweight class. The lowest AUC of 0.628 was observed in the male: $20-30$ group. Specific experiment results of all groups are described in Table 4.

The confusion matrix (also called a contingency table) in Table 5 describes more detailed performances of 6 models according to age and gender. For example, the classification model of the female: 20-30 group correctly predicted that
337 of 364 subjects with actual normal weight belonged to the "normal" class and that 30 of 133 subjects with actual overweight belonged to the "overweight" class. Moreover, the female: 40-50 model correctly predicted that 103 of 201 subjects with actual normal weight belonged to the "normal" class and that 168 of 244 subjects with actual overweight belonged to the "overweight" class.

Our experiments show that classification of normal and overweight status in the female: $40-50$ and male: $20-30$ groups was slightly difficult, compared with the other 4 groups and that classification of normal status and overweight status in the female: $20-30$ and female: 60 groups was superior compared with the other groups. The classification performance with wrapper-based feature selection was better than that without feature selection. Many of features selected by feature selection differed according to age- and genderspecific groups (see Table 3 ).

3.2. Statistical Analysis of Features Associated with Normal Weight and Overweight. The statistical data are expressed as mean \pm standard deviation. Comparisons between normal and overweight groups were performed using independent two-sample $t$-tests, and the $P$ values were adjusted using the Benjamin-Hochberg method to control the false discovery rate; $P$ values $<0.05$ and adjusted $P$ values $<0.05$ were considered statistically significant. Only statistically significant features among all features selected by wrapper-based feature subset selection in each group are described in Table 6. All statistical analyses were conducted using SPSS Statistics 19 and $\mathrm{R}$ package 2.15.0 for Windows.

In the female 20-30 group, 7 features were significantly different between the normal and the overweight classes $(P<0.05$ and adjusted $P<0.05)$. In this group, aF60_120_F240_480, aF240_480_960_1960, aF60_120_ 960_1960, and eF240_480_960_1960 (features related to the ratios of energies) were significantly different between the 2 classes $(P<0.001$, adjusted $P=0.005 ; P<0.001$, adjusted $P=0.005 ; P<0.001$, adjusted $P=0.005$; and $P<0.001$, adjusted $P=0.01$, resp.). These results indicate that the ratios of voice energies over the fixed frequency band in normal subjects are higher than those of the overweight subjects in this group. There were statistically significant differences with respect to eMFCC 4 and oMFCC 4 between the 2 classes $(P<0.05$, adjusted $P<0.05$; and $P<0.01$, adjusted $P<0.05$, resp.); particularly, the MFCC4 of vowel E and MFCC4 of vowel $\mathrm{O}$ of normal subjects $(1.277 \pm 6.836$ and $-4.087 \pm 5.624$, resp.) were higher than those of overweight subjects $(-0.801 \pm 8.315$ and $-5.989 \pm 7.191$, resp. $)$ in this group. In addition, SITS was significantly different between the 2 classes $(P<0.005$, adjusted $P=0.01)$. This result indicates that the average intensity of sentences in normal subjects $(56.14 \pm 7.515)$ is higher than that of overweight subjects (53.73 \pm 8.074$)$ among females aged $20-39$ years.

In the male 20-30 group, one eMFCC4 feature was significantly different between the normal and the overweight classes $(P<0.001$, adjusted $P<0.01)$. The MFCC4 of vowel $\mathrm{E}$ in normal subjects was higher than that of overweight subjects in this group. None of the features were significantly different within the other groups. 
TABLE 5: Confusion matrix (also called contingency table or error matrix) of 6 models according to age and gender in classification experiments with feature selection.

\begin{tabular}{|c|c|c|c|c|c|}
\hline \multirow{3}{*}{ Group } & \multicolumn{4}{|c|}{ Classification model $^{\mathrm{a}}$} & \\
\hline & Actual & \multicolumn{2}{|c|}{ Predicted } & \multicolumn{2}{|c|}{ Subjects $^{\mathrm{b}}$} \\
\hline & & Overweight & Normal & Overweight & Normal \\
\hline \multirow{2}{*}{ Female: $20-30$} & Overweight & 30 & 103 & \multirow{2}{*}{133} & \multirow{2}{*}{364} \\
\hline & Normal & 27 & 337 & & \\
\hline \multirow{2}{*}{ Female: $40-50$} & Overweight & 168 & 76 & \multirow{2}{*}{244} & \multirow{2}{*}{201} \\
\hline & Normal & 98 & 103 & & \\
\hline \multirow{2}{*}{ Female: 60} & Overweight & 86 & 18 & \multirow{2}{*}{104} & \multirow{2}{*}{41} \\
\hline & Normal & 26 & 15 & & \\
\hline \multirow{2}{*}{ Male: $20-30$} & Overweight & 139 & 67 & \multirow{2}{*}{206} & \multirow{2}{*}{175} \\
\hline & Normal & 84 & 91 & & \\
\hline \multirow{2}{*}{ Male: $40-50$} & Overweight & 154 & 25 & \multirow{2}{*}{179} & \multirow{2}{*}{77} \\
\hline & Normal & 48 & 29 & & \\
\hline \multirow{2}{*}{ Male: 60} & Overweight & 54 & 17 & \multirow{2}{*}{71} & \multirow{2}{*}{35} \\
\hline & Normal & 20 & 15 & & \\
\hline
\end{tabular}

${ }^{\mathrm{a}}$ Results of confusion matrix by classification model; ${ }^{\mathrm{b}}$ number of subjects of each class (overweight and normal) in original data.

TABLE 6: Statistical analysis results by independent two sample $t$-test and Benjamin-Hochberg's method.

\begin{tabular}{|c|c|c|c|c|c|c|c|}
\hline Group & Feature & Class & Mean & Std. & $T$ & $P$ value & Adj. $P$ value \\
\hline \multirow{14}{*}{ Female: $20-30$} & \multirow{2}{*}{ aF60_120_F240_480 } & Normal & 0.834 & 0.390 & \multirow{2}{*}{3.474} & \multirow{2}{*}{$<0.001$} & \multirow{2}{*}{0.005} \\
\hline & & Overweight & 0.699 & 0.365 & & & \\
\hline & \multirow{2}{*}{ aF240_480_960_1960 } & Normal & 2.285 & 0.818 & \multirow{2}{*}{3.510} & \multirow{2}{*}{$<0.001$} & \multirow{2}{*}{0.005} \\
\hline & & Overweight & 1.996 & 0.806 & & & \\
\hline & \multirow{2}{*}{ aF60_120_960_1960 } & Normal & 2.135 & 1.416 & \multirow{2}{*}{3.618} & \multirow{2}{*}{$<0.001$} & \multirow{2}{*}{0.005} \\
\hline & & Overweight & 1.631 & 1.248 & & & \\
\hline & \multirow{2}{*}{ eF240_480_960_1960 } & Normal & 3.033 & 0.627 & \multirow{2}{*}{3.342} & \multirow{2}{*}{$<0.001$} & \multirow{2}{*}{$<0.01$} \\
\hline & & Overweight & 2.818 & 0.660 & & & \\
\hline & \multirow{2}{*}{ eMFCC4 } & Normal & 1.277 & 6.836 & \multirow{2}{*}{2.581} & \multirow{2}{*}{$<0.05$} & \multirow{2}{*}{$<0.05$} \\
\hline & & Overweight & -0.801 & 8.315 & & & \\
\hline & \multirow{2}{*}{ oMFCC4 } & Normal & -4.087 & 5.624 & \multirow{2}{*}{2.757} & \multirow{2}{*}{$<0.01$} & \multirow{2}{*}{$<0.05$} \\
\hline & & Overweight & -5.989 & 7.191 & & & \\
\hline & \multirow{2}{*}{ SITS } & Normal & 56.14 & 7.515 & \multirow{2}{*}{3.106} & \multirow{2}{*}{$<0.005$} & \multirow{2}{*}{0.01} \\
\hline & & Overweight & 53.73 & 8.074 & & & \\
\hline \multirow{2}{*}{ Male: $20-30$} & \multirow{2}{*}{ eMFCC4 } & Normal & 5.057 & 6.678 & \multirow[t]{2}{*}{3.393} & \multirow{2}{*}{$<0.001$} & \multirow{2}{*}{$<0.01$} \\
\hline & & Overweight & 2.679 & 6.929 & & & \\
\hline
\end{tabular}

$P$ value $<0.05$ was considered statistically significant. The $P$ values were adjusted using the Benjamin-Hochberg method to control the false discovery rate. Only statistically significant features among all features selected by wrapper-based feature subset selection in each group are described in this table (Std: standard deviation, Adj: adjusted).

Despite the high accuracy and AUC of classification in the female $\geq 60$ group, no statistically significant differences were detected between the normal and overweight classes. Furthermore, we did not find features with a broad range of applicability for classifying the normal and overweight statuses in the age-or gender-specific classifications. We will discuss these problems further in Section 3.4.

3.3. Scalability and Applications. Some studies on patient BMI and weight estimation have focused on emergency medical services and telemedicine because the precise estimation of weight and BMI status in emergency medical care is very important for accurate counter-shock voltage calculation, drug dosage estimation, intensive care, and elderly trauma management [29, 54-57]. Although some issues must be addressed for accurate prediction of the BMI status, our method may have potential applications in telemedicine, remote healthcare, and real-time monitoring services to monitor the BMI status of patients with long-term obesityrelated diseases. Additionally, our method can be applied in the diagnosis of individual constitution types in remote healthcare. Pham et al. suggested that the BMI and cheek-tojaw width ratio were the most important predictive factors 
for the TaeEum (TE) constitution type [58], and Chae et al. proposed that the TE type tends to have a higher BMI than other types [59]. Furthermore, several studies mentioned that constitution types differed in speech features and body shape (BMI) [60-62]. Thus, through more studies on voice signals, $\mathrm{u}$-healthcare, body shape, and constitutions, the proposed classification method for BMI can be used to diagnose a constitution for personalized medical care, as the BMI is important in both alternative and Western medicines.

3.4. Limitations and Future Work. In our study, voice data of subjects were collected by a recording equipment in hospital site and research center site. In order to apply real-time diagnosis in telemedicine or $\mathrm{u}$-healthcare system, additional and important studies such as noise filter, adjustment technique, and handling of atypical speech in emergency, should be performed because of noise or interference generated by network or equipment during telecommunication.

Our method classified only normal and overweight classes and used voice data collected only from Korea. So, in order to more accurately classify a broad range of classessuch as underweight, normal, overweight, obese 1, obese 2, and obese 3-according to WHO standard classification in various ethnic groups, we must collect more and varied data sets.

In our classification experiments, the AUC with feature selection in the female $\geq 60$ group was the highest among all groups, although there were no significantly different features between the 2 classes among surviving features from the feature subset selection in the female $\geq 60$ group. We consider 2 aspects that could be responsible for the occurrence of this problem. First, this could be due to a combination problem of features in wrapper-based feature subset selection and classification problems. From the perspective of machine learning and data mining, machine learning for wrapper-based feature selection is considered a perfect black box. In general, greater numbers of features exhibiting significant differences lead to better machine-learning performance. However, we cannot guarantee that a classification using only significant features (i.e., those with $P$ values $<0.05$ ) always performs better than one using a combination of significant and less significant features. Therefore, the most important factor is the selection and combination of the features of each group. For example, Guyon and Elisseeff [43] suggest that the performance of variables that are ineffective by themselves can be improved significantly when combined with others. Furthermore, adding presumably redundant variables can result in noise reduction and consequently better class separation. The other possible reason for the observed problem is the lack of samples, which can force under- or overfitting in machine learning. The small sample size is a critical limitation of this study, because our sample size was not representative of the population. Thus, this study should be designated as a pilot observational study. In order to reduce or understand this problem, we require more samples and are currently collecting more samples.

In the future, we will investigate the extraction of useful features that demonstrate statistical significance in all ageand gender-specific groups, build a more accurate classification model, and collect more data for better classification performance. Furthermore, we will examine the association of the BMI with features such as respiration rate from nonstructured speech signals using a new protocol.

\section{Conclusions}

The classification of normal and overweight according to body mass index (BMI) is only possible through the measurement and calculation of weight and height. This study suggested a novel method for BMI classification by speech signal and showed the possibility of predicting a diagnosis of normal status or overweight status on the basis of voice and machine learning. We found discriminatory feature subsets for diagnosing normal or overweight individuals by feature selection. We proved that several features have a statistically significant difference between normal and overweight classes in the female: $20-30$ group and male: $20-30$ group through statistical analysis of the features selected by feature selection in each group. Our findings showed the possibility to predict BMI diagnosis using a combination of voice features without additional weight and height measurements, even if significant features are rare and weak. The prediction performance with feature selection was higher than that without feature selection. However, the accuracy and AUC achieved by our classification experiment were not yet sufficient for rigorous diagnosis and medical purposes. Therefore, we need more research about discriminatory features of broad range, rich data, and a more accurate classification model.

\section{Acknowledgment}

This work was supported by the National Research Foundation of Korea (NRF) Grant funded by the Korea government (MEST) (20120009001, 2006-2005173).

\section{References}

[1] T. J. Parsons, O. Manor, and C. Power, "Physical activity and change in body mass index from adolescence to midadulthood in the 1958 British cohort," International Journal of Epidemiology, vol. 35, no. 1, pp. 197-204, 2006.

[2] H. Hirose, T. Takayama, S. Hozawa, T. Hibi, and I. Saito, "Prediction of metabolic syndrome using artificial neural network system based on clinical data including insulin resistance index and serum adiponectin," Computers in Biology and Medicine, vol. 41, pp. 1051-1056, 2011.

[3] D. Gallagher, M. Visser, D. Sepúlveda, R. N. Pierson, T. Harris, and S. B. Heymsfieid, "How useful is body mass index for comparison of body fatness across age, sex, and ethnic groups?" American Journal of Epidemiology, vol. 143, no. 3, pp. 228-239, 1996.

[4] E. Anuurad, K. Shiwaku, A. Nogi et al., "The new BMI criteria for Asians by the regional office for the western pacific region of WHO are suitable for screening of overweight to prevent metabolic syndrome in elder Japanese workers," Journal of Occupational Health, vol. 45, no. 6, pp. 335-343, 2003.

[5] L. L. Yan, M. L. Daviglus, K. Liu et al., "BMI and health-related quality of life in adults 65 years and older," Obesity Research, vol. 12, no. 1, pp. 69-76, 2004. 
[6] Asia Pacific Cohort Studies Collaboration, "Body mass index and cardiovascular disease in the Asia-Pacific Region: an overview of 33 cohorts involving 310000 participants," International Journal of Epidemiology, vol. 33, pp. 751-758, 2004.

[7] C. M. Lee, S. Colagiuri, M. Ezzati, and M. Woodward, "The burden of cardiovascular disease associated with high body mass index in the Asia-Pacific region," Obesity Reviews, vol. 12, pp. e454-e459, 2011.

[8] L. Li, A. P. De Moira, and C. Power, "Predicting cardiovascular disease risk factors in midadulthood from childhood body mass index: utility of different cutoffs for childhood body mass index," American Journal of Clinical Nutrition, vol. 93, no. 6, pp. 1204-1211, 2011.

[9] H. S. Park, Y. S. Yun, J. Y. Park, Y. S. Kim, and J. M. Choi, "Obesity, abdominal obesity, and clustering of cardiovascular risk factors in South Korea," Asia Pacific Journal of Clinical Nutrition, vol. 12, no. 4, pp. 411-418, 2003.

[10] J. Y. Kim, H. M. Chang, J. J. Cho, S. H. Yoo, and S. Y. Kim, "Relationship between obesity and depression in the Korean working population," Journal of Korean Medical Science, vol. 25, no. 11, pp. 1560-1567, 2010.

[11] H. Fonseca, A. M. Silva, M. G. Matos et al., "Validity of BMI based on self-reported weight and height in adolescents," Acta Paediatrica, vol. 99, pp. 83-88, 2010.

[12] Y. F. Liao, M. L. Chuang, C. S. Huang, and Y. Y. Tsai, "Upper airway and its surrounding structures in obese and nonobese patients with sleep-disordered breathing," Laryngoscope, vol. 114, no. 6, pp. 1052-1059, 2004.

[13] W. T. Fitch and J. Giedd, "Morphology and development of the human vocal tract: a study using magnetic resonance imaging," Journal of the Acoustical Society of America, vol. 106, no. 3, pp. 1511-1522, 1999.

[14] S. Evans, N. Neave, and D. Wakelin, "Relationships between vocal characteristics and body size and shape in human males: an evolutionary explanation for a deep male voice," Biological Psychology, vol. 72, no. 2, pp. 160-163, 2006.

[15] N. J. Lass, "Correlational study of speakers' heights, weights, body surface areas, and speaking fundamental frequencies," Journal of the Acoustical Society of America, vol. 63, no. 4, pp. 1218-1220, 1978.

[16] N. J. Lass, J. K. Phillips, and C. A. Bruchey, "The effect of filtered speech on speaker height and weight identification," Journal of Phonetics, vol. 8, pp. 91-100, 1980.

[17] W. A. van Dommelen and B. H. Moxness, "Acoustic parameters in speaker height and weight identification: sex-specific behaviour," Language and speech, vol. 38, no. 3, pp. 267-287, 1995.

[18] J. González, "Formant frequencies and body size of speaker: a weak relationship in adult humans," Journal of Phonetics, vol. 32, no. 2, pp. 277-287, 2004.

[19] H. J. Kunzel, "How well does average fundamental frequency correlate with speaker height and weight?" Phonetica, vol. 46, no. 1-3, pp. 117-125, 1989.

[20] D. G. Childers and K. Wu, "Gender recognition from speech. Part II: fine analysis," Journal of the Acoustical Society of America, vol. 90, pp. 1841-1856, 1991.

[21] L. Bruckert, J. S. Liénard, A. Lacroix, M. Kreutzer, and G. Leboucher, "Women use voice parameters to assess men's characteristics," Proceedings of the Royal Society, vol. 273, no. 1582, pp. 83-89, 2006.
[22] P. Belin, S. Fecteau, and C. Bédard, "Thinking the voice: neural correlates of voice perception," Trends in Cognitive Sciences, vol. 8, no. 3, pp. 129-135, 2004.

[23] M. Jessen, "Speaker classification in forensic phonetics and acoustics," in Speaker Classification I, pp. 180-204, Springer, Berlin, Germany, 2007.

[24] I. Mporas and T. Ganchev, "Estimation of unknown speaker's height from speech," International Journal of Speech Technology, vol. 12, no. 4, pp. 149-160, 2009.

[25] J. Gonzalez, 'Estimation of speakers' weight and height from speech: a re-analysis of data from multiple studies by lass and colleagues," Perceptual and Motor Skills, vol. 96, no. 1, pp. 297304, 2003.

[26] R. Greisbach, "Estimation of speaker height from formant frequencies," Forensic Linguistics, vol. 6, no. 2, pp. 265-277, 1999.

[27] G. Muhammad, T. A. Mesallam, K. H. Malki, M. Farahat, M. Alsulaiman, and M. Bukhari, "Formant analysis in dysphonic patients and automatic Arabic digit speech recognition," BioMedical Engineering OnLine, vol. 10, article 41, 2011.

[28] K. Umapathy and S. Krishnan, "Feature analysis of pathological speech signals using local discriminant bases technique," Medical and Biological Engineering and Computing, vol. 43, no. 4, pp. 457-464, 2005.

[29] W. L. Hall, G. L. Larkin, M. J. Trujillo, J. L. Hinds, and K. A. Delaney, "Errors in weight estimation in the emergency department: comparing performance by providers and patients," Journal of Emergency Medicine, vol. 27, no. 3, pp. 219-224, 2004.

[30] R. S. Lin, S. D. Horn, J. F. Hurdle, and A. S. GoldfarbRumyantzev, "Single and multiple time-point prediction models in kidney transplant outcomes," Journal of Biomedical Informatics, vol. 41, no. 6, pp. 944-952, 2008.

[31] G. D. Fiol and P. J. Haug, "Classification models for the prediction of clinicians' information needs," Journal of Biomedical Informatics, vol. 42, no. 1, pp. 82-89, 2009.

[32] A. de Leiva, M. E. Hernando, M. Rigla et al., "Telemedical artificial pancreas: PARIS (Pancreas Artificial Telemedico Inteligente) research project," Diabetes Care, vol. 32, pp. S211216, 2009.

[33] J. M. Wojcicki, P. Ladyzynski, J. Krzymien et al., "What we can really expect from telemedicine in intensive diabetes treatment: results from 3-year study on type 1 pregnant diabetic women," Diabetes Technology and Therapeutics, vol. 3, no. 4, pp. 581-589, 2001.

[34] W. Y. Jen, "The adoption of mobile weight management services in a virtual community: the perspective of college students," Telemedicine and e-Health, vol. 16, no. 4, pp. 490-497, 2010.

[35] J. R. Warren, K. J. Day, C. Paton et al., "Implementations of health information technologies with consumers as users: findings from a systematic review," Health Care and Informatics Review Online, vol. 14, no. 3, pp. 2-17, 2010.

[36] M. J. Morón, A. Gómez-Jaime, J. R. Luque, and E. Casilari, "Development and evaluation of a Python telecare system based on a Bluetooth Body Area Network," Eurasip Journal on Wireless Communications and Networking, vol. 2011, Article ID 629526, 2011.

[37] A. C. Norris, "Scope, Benefits and limitations of telemedicine," in Essentials of Telemedicine and Telecare, pp. 30-35, John Wiley \& Sons, Chichester, UK, 2002.

[38] K. H. Kim, B. Ku, S. Kang, Y. S. Kim, J. S. Jang, and J. Y. Kim, "Study of a vocal feature selection method and vocal properties for discriminating four constitution types," Evidence-Based 
Complementary and Alternative Medicine, vol. 2012, Article ID 831543, 10 pages, 2012.

[39] WHO Expert Consultation, "Appropriate body-mass index for Asian populations and its implications for policy and intervention strategies," The Lancet, vol. 363, pp. 157-163, 2004.

[40] T. Haas, S. Svacina, J. Pav, R. Hovorka, P. Sucharda, and J. Sonka, "Risk calculation of type 2 diabetes," Computer Methods and Programs in Biomedicine, vol. 41, no. 3-4, pp. 297-303, 1994.

[41] K. K. Khokhar, G. Kaur, and S. Sidhu, "Prevalence of obesity in working premenopausal and postmenopausal women of Jalandhar District, Punjab," Journal of Human Ecology, vol. 29, pp. 57-62, 2010.

[42] World Health Organisation, The Asia-Pacific Perspective: Redefining Obesity and Its Treatment, Health Communications, Sydney, Australia, 2000.

[43] I. Guyon and A. Elisseeff, "An introduction to variable and feature selection," Journal of Machine Learning Research, vol. 3, pp. 1157-1182, 2003.

[44] R. Kohavi and G. H. John, "Wrappers for feature subset selection," Artificial Intelligence, vol. 97, no. 1-2, pp. 273-324, 1997.

[45] S. le Cessie and J. C. van Houwelingen, "Ridge estimators in logistic regression," Applied Statistics, vol. 41, no. 1, pp. 191-201, 1992.

[46] H. Ian, Data Mining: Practical Machine Learning Tools and Techniques, Morgan Kaufmann, San Francisco, Calif, USA, 2nd edition, 2005.

[47] J. Han and M. Kamber, Data Mining: Concepts and Techniques, Morgan Kaufmann, San Francisco, Calif, USA, 2nd edition, 2006.

[48] B. J. Lee, M. S. Shin, Y. J. Oh, H. S. Oh, and K. H. Ryu, "Identification of protein functions using a machine-learning approach based on sequence-derived properties," Proteome Science, vol. 7, article 27, 2009.

[49] P. N. Tan, M. Steinbach, and V. Kumar, Introduction to Data Mining, Addison Wesley, Boston, Mass, USA, 2006.

[50] J. Huang and C. X. Ling, "Using AUC and accuracy in evaluating learning algorithms," IEEE Transactions on Knowledge and Data Engineering, vol. 17, pp. 299-310, 2005.

[51] D. J. Hand, "Evaluating diagnostic tests: the area under the ROC curve and the balance of errors," Statistics in Medicine, vol. 29, no. 14, pp. 1502-1510, 2010.

[52] C. E. Metz, "ROC analysis in medical imaging: a tutorial review of the literature," Radiological physics and technology, vol. 1, no. 1, pp. 2-12, 2008.

[53] R. Kumar and A. Indrayan, "Receiver operating characteristic (ROC) curve for medical researchers," Indian Pediatrics, vol. 48, no. 4, pp. 277-287, 2011.

[54] D. Krieser, K. Nguyen, D. Kerr, D. Jolley, M. Clooney, and A. M. Kelly, "Parental weight estimation of their child's weight is more accurate than other weight estimation methods for determining children's weight in an emergency department?" Emergency Medicine Journal, vol. 24, no. 11, pp. 756-759, 2007.

[55] T. R. Coe, M. Halkes, K. Houghton, and D. Jefferson, "The accuracy of visual estimation of weight and height in preoperative supine patients," Anaesthesia, vol. 54, no. 6, pp. 582586, 1999.

[56] S. Menon and A. M. Kelly, "How accurate is weight estimation in the emergency department?" Emergency Medicine Australasia, vol. 17, no. 2, pp. 113-116, 2005.
[57] R. J. Moran, R. B. Reilly, P. De Chazal, and P. D. Lacy, "Telephony-based voice pathology assessment using automated speech analysis," IEEE Transactions on Biomedical Engineering, vol. 53, no. 3, pp. 468-477, 2006.

[58] D. D. Pham, J. H. Do, B. Ku, H. J. Lee, H. Kim, and J. Y. Kim, "Body mass index and facial cues in Sasang typology for young and elderly persons," Evidence-Based Complementary and Alternative Medicine, vol. 2011, Article ID 749209, 2011.

[59] H. Chae, I. K. Lyoo, S. J. Lee et al., "An alternative way to individualized medicine: psychological and physical traits of Sasang typology," Journal of Alternative and Complementary Medicine, vol. 9, no. 4, pp. 519-528, 2003.

[60] B. J. Lee, B. Ku, K. Park, K. H. Kim, and J. Y. Kim, "A new method of diagnosing constitutional types based on vocal and facial features for personalized medicine," Journal of Biomedicine and Biotechnology, vol. 2012, Article ID 818607, 2012.

[61] S. W. Lee, E. S. Jang, J. Lee, and J. Y. Kim, "Current researches on the methods of diagnosing sasang constitution: an overview," Evidence-based Complementary and Alternative Medicine, vol. 6, no. 1, pp. 43-49, 2009.

[62] J. H. Do, E. S. Jang, B. Ku, J. S. Jang, H. Kim, and J. Y. Kim, "Development of an integrated Sasang constitution diagnosis method using face, body shape, voice, and questionnaire information," BMC Complementary and Alternative Medicine, vol. 12, article 9, 2012. 


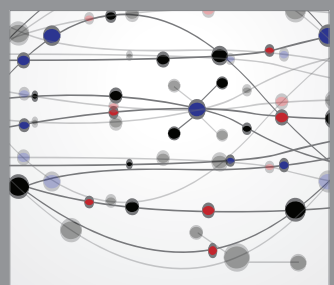

The Scientific World Journal
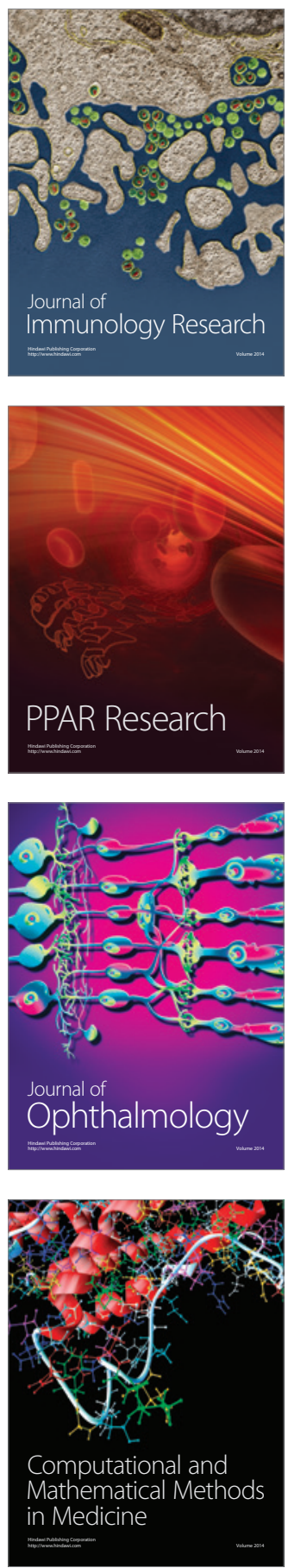

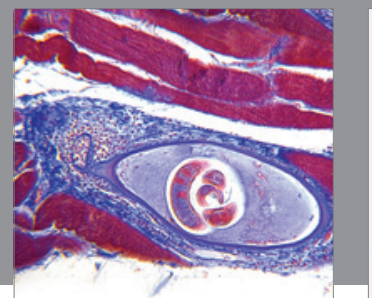

Gastroenterology

Research and Practice
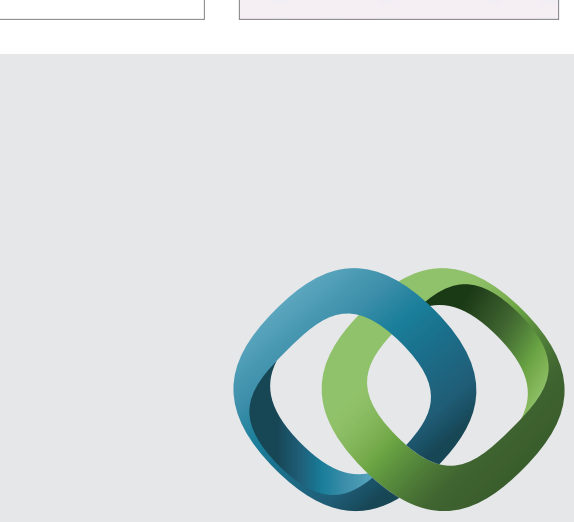

\section{Hindawi}

Submit your manuscripts at

http://www.hindawi.com
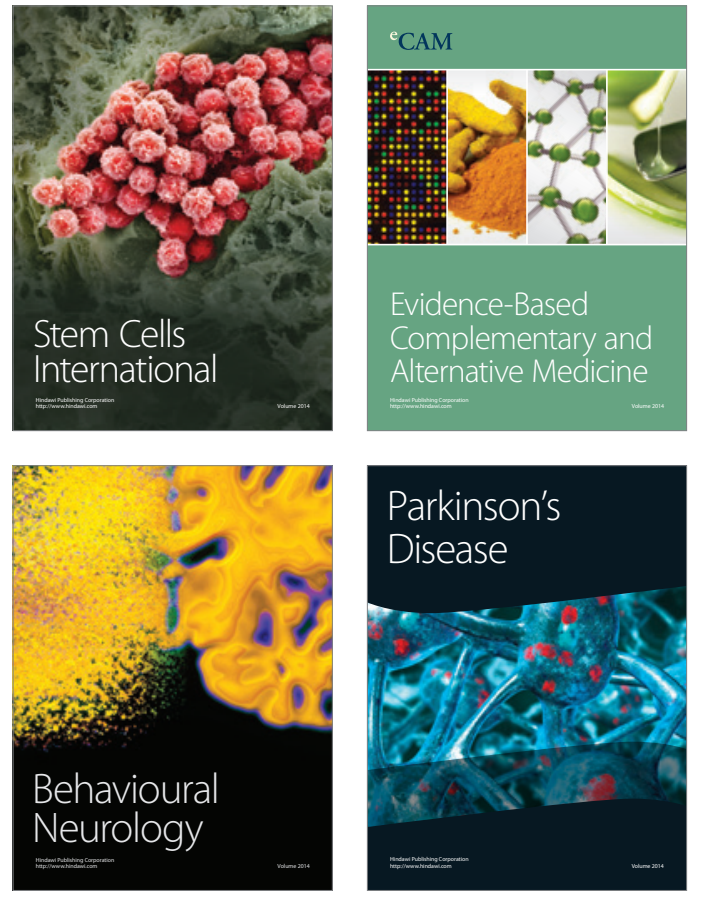
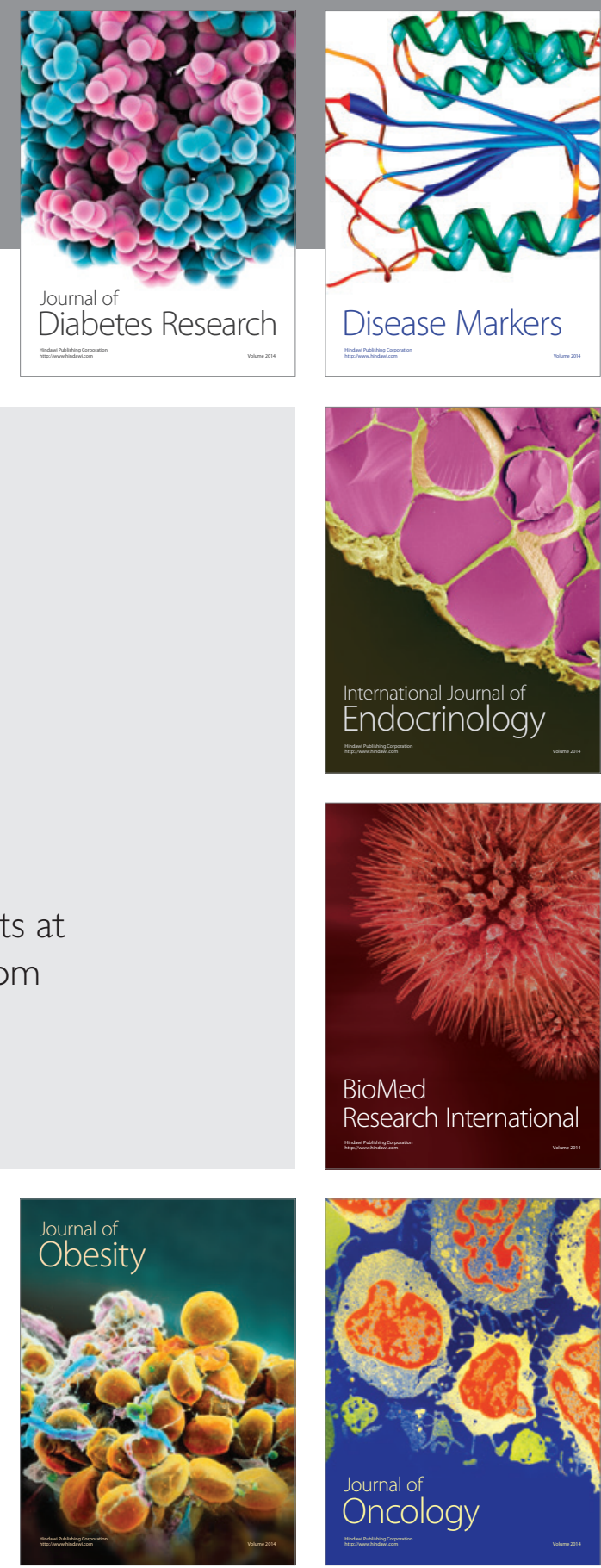

Disease Markers
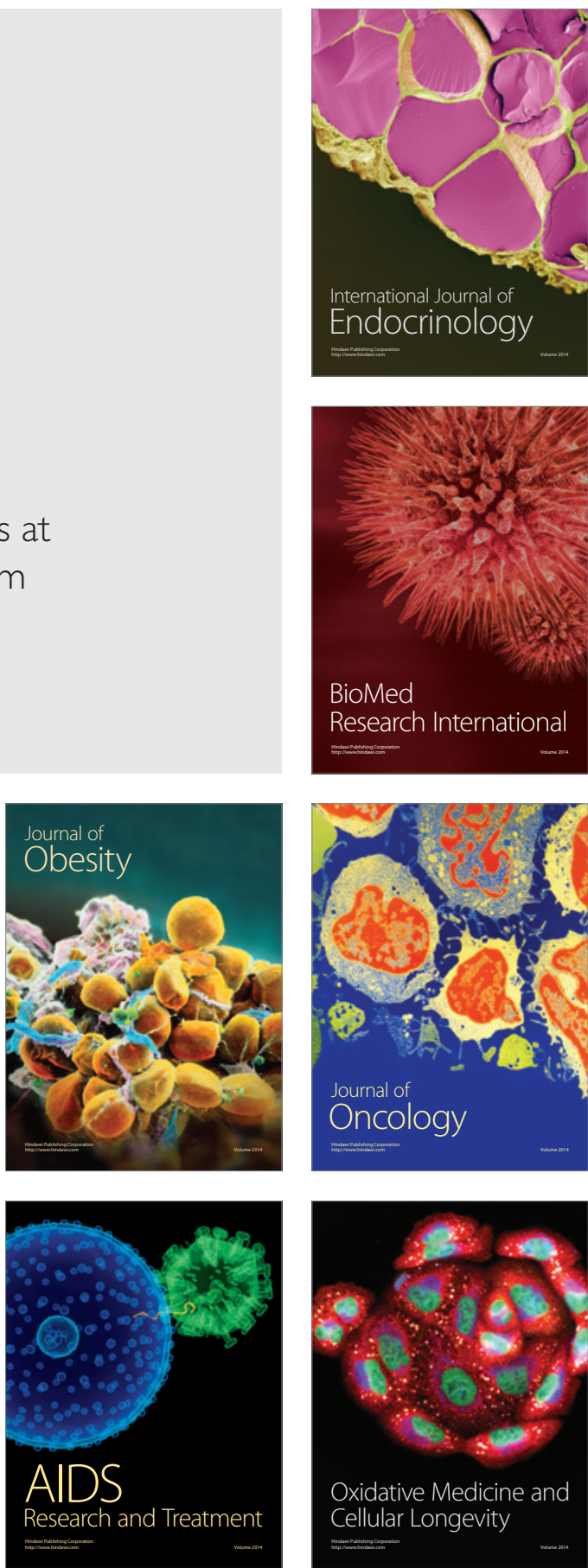